\title{
The intensity of emotion: Altered motor simulation impairs processing of facial
}

expressions in congenital facial palsy

\section{Arianna Schiano Lomoriello ${ }^{12}$, Giulio Caperna ${ }^{3}$, Elisa De Stefani ${ }^{4}$, Pier Francesco Ferrari ${ }^{*}$, \&} Paola Sessa ${ }^{1,6^{*}}$

${ }^{1}$ Department of Developmental and Social Psychology, University of Padova, Padova, Italy

${ }^{2}$ Department of Cognitive System, Denmark Technical University (DTU), Copenhagen, Denmark

${ }^{3}$ Joint Research Centre of the European Commission, Ispra, Italy

${ }^{4}$ Department of Medicine and Surgery, University of Parma, Parma, Italy

${ }^{5}$ Institut des Sciences Cognitives Marc Jeannerod, CNRS/Université Claude Bernard Lyon, France

${ }^{6}$ Padova Neuroscience Center (PNC), University of Padova, Padova, Italy

\section{*Co-Corresponding authors:}

Paola Sessa, Department of Developmental and Social Psychology, University of Padova, Via Venezia 8, 35121, Padua, Italy.

paola.sessa@unipd.it

Pier Francesco Ferrari, Institut des Sciences Cognitives Marc Jeannerod, Unit 5229, CNRS/ Université Claude Bernard Lyon, 67 Bd Pinel, 69675 Bron Cedex, France pierfrancesco.ferrari@isc.cnrs.fr 


\begin{abstract}
According to the models of sensorimotor simulation, we recognize others' emotions by subtly mimicking their expressions, which allows us to feel the corresponding emotion via facial feedback. In this contest, facial mimicry, which requires the implicit activation of the motor programs that produce a specific expression, is a crucial phenomenon occurring in emotion recognition, also concerning expression intensity. Consequently, difficulties to produce facial expressions would affect the experience of emotional understanding. In the present investigation, we recruited a sample $(\mathrm{N}=$ 11) of patients with Moebius syndrome (MBS), characterized by congenital facial paralysis, and a control group $(\mathrm{N}=11)$ of healthy participants. By leveraging the MBS unique condition, we aimed at investigating the role of facial mimicry and sensorimotor simulation in creating a precise embodied concept of each emotion. The two groups underwent a sensitive facial emotion recognition task, optimally tuned to test sensitivity to emotion intensity and emotion discriminability in terms of their confusability with other emotions. Our study provides evidence of a deficit in recognizing emotions in MBS patients, expressed by a significant decrease in the rating of the intensity of three specific emotion categories, namely sadness, fear and disgust. Moreover, we observed an impairment in detecting these emotions, resulting in a stronger confusability of such emotions with the neutral and the secondary blended emotion. These findings provide support for embodied theories, which hypothesize that sensorimotor systems are involved in the detection and discrimination of emotions.
\end{abstract}

Key words: motor simulation, facial expressions, emotion, Moebius syndrome, facial mimicry 


\section{Introduction}

The ability to express and recognize facial expressions is crucial for social interactions (Elfenbein, 2007). A long-standing question that has repeatedly interested and fascinated psychologists, neuroscientists and philosophers concerns the cognitive mechanisms and their neural implementation that allow visual experiences of others' facial expressions to be translated into visual experiences of others' emotions. This mechanism requires some form of mediation mechanism capable of transforming a visual pattern - like that of a facial expression - in an emotion felt by the other (observed) individual. Nonetheless, there is no unequivocal agreement regarding what kind of information is used and extracted to recognize another person's emotion (Gallese \& Goldman, 1998; Goldman \& Sripada, 2005; Hess \& Blairy, 2001; Niedenthal, Mermillod, Maringer, \& Hess, 2010; Orlowska, Krumhuber, Rychlowska, \& Szarota, 2018; Wood, Rychlowska, Korb, \& Niedenthal, 2016).

Different theories have tried to entail this question. According to one view a potential mechanism would require an in-depth visual analysis of the facial expression. In this vein, facial expression recognition would be exploited by inferential processes, based on a system of knowledge and beliefs (Gallese \& Goldman, 1998; Gopnik \& Wellman, 1992; Leslie, 1994), mainly referred as Theory of Mind (ToM, Fletcher et al., 1995; Frith \& Frith, 2005). Nevertheless, experimental evidence that newborns and infants mimic others' facial expressions and react to them accordingly with the emotions they convey, seems to contrast with this view; for instance, they react with avoidance and fear to negative expressions long before a sophisticated capacity for ToM has developed (Ruba, Meltzoff, \& Repacholi, 2019), suggesting that the potential process underlying understanding others' emotions is more likely based on early, pre-wired mechanisms rather than on high-level cognitive skills (Preston \& de Waal, 2002). An alternative mechanism to the ToM proposes that the recognition of others' emotions requires both visual analysis and 'sensorimotor simulation' (de la Rosa, Fademrecht, Bülthoff, Giese, \& Curio, 2018; Paracampo, Pirruccio, Costa, Borgomaneri, \& Avenanti, 2018) - an 
unconscious, covert imitation and automatic activation of the sensorimotor programs of the observed facial postures or movements (Goldman \& Sripada, 2005; Ipser \& Cook, 2016; Montgomery \& Haxby, 2008; Niedenthal, Mermillod, Maringer, \& Hess, 2010; Paracampo, Tidoni, Borgomaneri, Di Pellegrino, \& Avenanti, 2017), suggesting that humans are 'cabled' with a neural mechanism that let them to almost literally resonate with others (Adolphs, 2009; Ferrari, Tramacere, Simpson, \& Iriki, 2013; Gallese, 2016). This mechanism, in turn, would activate interconnected systems, including the limbic system, allowing observers to re-enact the others' affective states, and therefore, in last analysis, to understand them (Wood, Rychlowska, Korb, \& Niedenthal, 2016; Tramacere, Pievani, \& Ferrari, 2017). The most recent model of sensorimotor simulation of others' facial expressions has been proposed by Wood and colleagues (2016) and includes different stages of processing, also occurring in parallel and that would operate as follows: When an observer is exposed to a facial expression both the extrastriate regions for the visual analysis of faces/facial expressions and the sensorimotor cortices (i.e., premotor cortex, primary motor cortex and somatosensory areas) become activated. This distributed neural activity may or may not be accompanied by the reproduction, at a sub-threshold level, of the observed facial expression, i.e. a phenomenon known as 'facial mimicry', i.e. the spontaneous, automatic and unconscious activation of facial muscles that is elicited when an emotional expression is observed. A secondary activation would then spread to other brain regions, such as the limbic system and areas involved in emotional processing and in the control of the autonomic responses of the organism associated with somatic facial activity. This entire process would next lead to the experience of the emotion in the first person, involving cognitive, behavioral and physiological changes, up to the higher-level cognitive systems. The model predicts that the simulation can provide backward feedback to visual cortices by shaping the construction of visual percepts, possibly leading to more accurate visual representations, also with regard to expression intensity (Wood, Lupyan, Sherrin, \& Niedenthal, 2016). 
Three main types of evidence are typically cited in support of this simulation mechanism in the process of recognizing others' emotions. First, patients with lesions of somatosensory, motor and/or premotor cortices show deficits in recognition of facial expressions (Adolph, 2002). Second, studies of facial expressions recognition in which transcranial magnetic stimulation (TMS) has been used to inhibit the activity of the cortical motor areas involved in the simulation have demonstrated decrements of participants' accuracy (Korb et al., 2016; Pitcher, Garrido, Walsh, \& Duchaine, 2008; Pourtois et al., 2004). The third line of evidence is strictly connected to the facial mimicry mechanism, demonstrating that both in case of blockage/alteration by means of various experimental manipulations (e.g. pens or chopsticks [Ponari, Conson, D’Amico, Grossi, \& Trojano, 2012], mouthguards [Rychlowska et al., 2014], hardening gels [Wood et al., 2016], botulinum [Baumeister, Papa, \& Foroni, 2016]) and in case of individuals suffering from congenital or acquired facial and expression production disorders, difficulties in recognizing facial expressions have been reported (Bate, Cook, Mole, \& Cole, 2013; Calder, Keane, Cole, Campbell, \& Young, 2000; Giannini et al., 1984; McKone \& Robbins, 2011; Nicolini et al., 2019; De Stefani et al. 2019). This whole body of evidence supports the notion that the simulation mechanism may optimally work when facial feedback is consistent with the internal simulation (Wolpert \& Flanagan, 2001); thus, interfering with facial mimicry may cause an incongruent signal with the pattern of sensorimotor activity and would result in a drop of recognition/discrimination accuracy (Wingenbach, Brosnan, Pfaltz, Plichta, \& Ashwin, 2018; Wood et al., 2016; Wood et al., 2016).

In this theoretical and empirical panorama, experimental investigations examining patients with facial palsy, especially congenital, are decisive. Indeed, testing patients with the inability to use facial mimicry from birth could enhance existing knowledge about the role of facial mimicry and sensorimotor simulation in the mechanism of immediate and automatic translation of others' facial expressions into others' 'emotions'. Importantly, such subjects with congenital facial palsy not only cannot use their facial mimicry, but they also cannot re-activate, at least complete, sensorimotor 
representations of facial expressions. Previous clinical evidence found an impairment in face recognition performance in both Parkinson and Huntington's disease patients compared to matched healthy controls (Ricciardi, Comandini, \& Erro, 2017; Trinkler et al. 2017; Gray \& Tickle-Degnen, 2010; Sprengelmeyer et al., 1996). However, it should be noted that these neurological diseases, which present facial amimia could exhibit other physical (e.g. rigidity, tremors, postural instability and reduced expressivity in the body and voice), and also psychological symptoms, such as depression, which may have a confounding effect on the decoding of facial affect (Tickle-Degnen, 2010). Consequently, these clinical populations do not allow to clarify the mere effect of the lack of facial mimicry on emotional processing. In this vein, we involved subjects with Moebius Syndrome (MBS) ${ }^{1}$, an extremely rare congenital non-progressive condition resulting in severe or complete bilateral facial paralysis, resulting in a selective alteration of facial mimicry. Given the rarity of the syndrome, only few studies have investigated emotion recognition in MBS subjects, and, further, they present inconsistent results (Bate et al., 2013; Bogart \& Matsumoto, 2010; Calder et al., 2000; Giannini et al., 1984; Nicolini et al., 2019; De Stefani et al., 2019).

Overall, a deficit - albeit mild - in the recognition of others' facial expressions seems observable when these are ambiguous or subtle (i.e. characterized by a low/moderate intensity of the emotion expressed). To note, this observation is currently based on the results of a very few studies, including a single case study using a task of facial expressions recognition of subjects involved in gambling (slot machines; Giannini et al., 1984), a study that involved three MBS subjects in a task of discrimination of ambiguous emotions (Exp. 2; Calder et al., 2000) and a study that included six MBS subjects in identification tasks of basic facial expressions (Exp. 1a), ambiguous facial expressions (Exp.

\footnotetext{
${ }^{1}$ Also called 'congenital facial diplegia' or 'congenital ocular-facial paralysis'. The incidence of this syndrome varies from 1 in 50,000 to 1 in 500,000 births (Rasmussen, Rian; O, Korshoej, \& Christensen, 2015), mainly affecting individuals of normal intelligence and cognitive development. The most evident characteristic of the syndrome is the unilateral or, more frequently, bilateral nonprogressive congenital facial palsy (VII cranial nerve) with impairments of ocular abduction (VI cranial nerve) (Abramson, Cohen, \& Mulliken, 1998; Bianchi, Copelli, Ferrari, Ferri, \& Sesenna, 2010; Cattaneo, Chierici, Bianchi, Sesenna, \& Pavesi, 2006).
} 
1b) and in a choice task of adjectives that best describes the emotional state of the observed model (Exp. 1c) (Bate et al., 2013). The study by Nicolini et al. (2019), instead, tested 9 MBS children (5.7 \pm 1.78 years) monitoring their autonomic activity by means of functional infrared thermal imaging technique in an emotion comprehension test. Interestingly, children with MBS showed weaker temperature changes (i.e. reduced autonomic activity) than controls when viewing emotional stimuli. Finally, De Stefani and colleagues (2019), conducted two experiments testing for emotional processing in children $(9 \pm 2.3)$ with MBS. In particular, the authors investigated both the ability to recognize dynamic facial expressions and the responses of the autonomic nervous system during emotional processing by comparing the MBS to a matched control group. Interestingly, this study showed that the MBS have a less responsive parasympathetic system during observation of dynamic social stimuli. Moreover, the authors found a mild deficit in emotion recognition by comparing the MBS to the control group. On the contrary, few studied reported as MBS subjects did not differ from the matched control group or normative data in facial expression recognition accuracy (Bate et al., 2013; Calder et al., 2000; Bogart \& Matsumoto, 2010), leading to the conclusion that facial mimicry and facial feedback are not necessary to recognize facial expressions and that accuracy was not related to the MBS ability to produce facial expressions in the first person. With a certain degree of agreement, researchers in this field, embracing a neuroconstructivist perspective, proposed the idea that MBS individuals, who have lived without the ability to mimic facial expressions for their entire lives, have developed compensatory mechanisms for the role played by facial mimicry/simulation in emotion recognition and do not use the same neural pathways as typical developed individuals (McIntosh, Reichmann-Decker, Winkielman, \& Wilbarger, 2006; De Stefani et al. 2019).

Furthermore, a potential explanation of the discrepant results in this field suggests that facial mimicry, and consequently sensorimotor simulation, may contribute to facial expression recognition efficiency particularly when the tasks are more challenging, such as when facial expressions are more complex, must be interpreted quickly, only partial information is available, or when the task requires 
subtle discriminations such as discriminating fake versus genuine smiles (Niedenthal et al., 2010; Paracampo et al., 2017). However, it is legitimate to hypothesize that if the observer's facial mimicry has a crucial role in the dynamic modeling of the percept - as hypothesized by the model of Wood and colleagues (2016) - we should still expect that expressions characterized by different intensities are perceived differently in individuals with Moebius Syndrome when compared to healthy control participants. This hypothesis seems to be supported by recent studies using the event-related potential technique (ERP) to monitor the impact of blocking the observer's facial expressions (via gel mask) on the online processing of facial expressions (Schiano Lomoriello, Maffei, Brigadoi, \& Sessa, 2019; Sessa, Schiano Lomoriello, \& Luria, 2018). These studies have demonstrated how both early, i.e. P1 component (Schiano Lomoriello et al., 2019), and later stages of face processing, i.e. as suggested by the modulation of the amplitude of the SPCN ERP component - an index of the precision of visual working memory (VWM) representation (Sessa et al., 2018) - are modulated as a function of participants' mimicry manipulation. These findings actively support the hypothesis of a role of facial mimicry on the construction of perceptual/VWM representations of facial expressions of others' emotions (see also Gambarota \& Sessa, 2019).

In the present investigation, we used a behavioral task originally implemented by Heberlein, Padon, Gillihan, Farah and Fellows (2008), in which the authors wanted to provide a precise characterization of the role of human ventromedial prefrontal cortex in facial emotion recognition. Briefly, the task involved evaluating the intensity of each of six emotions (i.e. anger, disgust, fear, happiness, sadness, surprise) for each face of a set of expressions of different intensity. So, for example, the subjects had to evaluate the intensity of happiness perceived for each of the faces of the set, both for those who had a (more or less intense) expression of happiness and for those showing facial expressions other than happiness. In the present study, our goal was to investigate the role of facial mimicry and sensorimotor simulation in evaluating emotions in facial expressions by comparing the 
judgements of a group of MBS to a group of healthy participants matched for age and gender. By leveraging the unique condition of MBS subjects, we aimed at clarifying whether mimicry and sensorimotor simulation may contribute, not only in recognizing an emotion among others (i.e. emotion categorization), which could also be done via a compensatory mechanism, but in creating a precise embodied concept of each emotion. In particular, this study aimed at understanding whether facial motor deficits could affect the judgement of intensity of the observed emotions enhancing our knowledge on the general functioning of facial mimicry and simulative processes, providing a picture of how they contribute in categorizing each emotion in relation to other emotions.

Although there is evidence of how the discrimination of facial expressions can be described by different activations of sets of face areas (Schyns, Bonnar, \& Gosselin, 2002), however, it is possible to observe similarities in the muscle activation patterns of some emotions, explaining why an observer could confuse one emotion with another by observing a certain facial expression. In this framework, the role of sensorimotor simulation is still unknown. For example, it has been proved that happiness is recognized the best and confused the least (Palermo \& Coltheart, 2004), whilst other emotions, such as anger and disgust (Pochedly, Widen, \& Russell, 2012) or fear and surprise (Palermo \& Coltheart, 2004) are frequently confused and also conceptualized as similar in subjective experiences (Plutchik, 1997).

We also wanted to track the role of mimicry and sensorimotor simulation in building this concept of each emotion and for this purpose, we used the same task implemented by Heberlein and colleagues (2008), which is optimally tuned to test sensitivity to emotion intensity and emotion discriminability in terms of their confusability with other emotions. Indeed, the task is able to provide key information on participants' ability to detect an emotion - expressed by the difference between the coherent emotion to be evaluated and the neutral one - and to rate it as absent when absent - which mostly rely on the ability to identify the primary emotion category among the others correctly. 
Starting with these considerations, we hypothesize to observe a difference between the two groups in the intensity assigned to each emotion category. Furthermore, we hypothesized that Moebius subjects could more easily confuse one emotion with another than the subjects in the control group. Given the heterogeneity of the syndrome (Briegel, 2006), which may cause a diverse impairment in different emotion categories, we did not have specific hypotheses on which emotion could have resulted as more impacted compared to healthy individuals.

\section{Material and Methods}

\subsection{Participants}

The study involved 22 participants. Eleven adults with MBS (MBS group, MBS 6 females and 5 males, $M_{\text {age }}=35,66$ years; $\left.\mathrm{SD}=15,31\right)$ were recruited through the Italian Association of Moebius Syndrome (AISMo) and the Operative Unit of Maxillo-Facial Surgery Head and Neck Department, of the University of Parma. Data collection was conducted at the University of Padua and also in Assisi during the annual national meeting of the AISMo. In Table 1, demographic data and clinical information concerning all participants with MBS are reported. The inclusion criteria for children with MBS were (1) a certified diagnosis of unilateral or bilateral facial paralysis (Terzis \& Noah, 2003). Exclusion criteria were (1) the presence of congenital limb malformations and (2) the presence of any psychiatric or physical illness at the time of participation. Eleven healthy adults were also recruited as a control group matched in terms of age and gender with the MBS group. The participants of the control group reported normal or corrected to normal vision through the use of lenses or glasses and did not have intellectual disabilities or other psychological or neuropsychological disorders at the time of the test. All participants gave their informed written consent after full explanation of the procedure, which is in accordance with the Declaration of Helsinki. The study was approved by the Ethics Committee of the University of Padova (Protocol number 2806). 


\begin{tabular}{|c|c|c|c|c|c|}
\hline Subject & Age & Gender & Paralysis & Cranial nerves involved & Disfunction \\
\hline \multirow{2}{*}{ MBS1 } & \multirow{2}{*}{52} & \multirow{2}{*}{ Male } & \multirow{2}{*}{ Bilateral } & Abducens Nerve (VI) & No lateral eye movements \\
\hline & & & & Facial Nerve (VII) & Facial palsy \\
\hline \multirow{2}{*}{ MBS2 } & \multirow{2}{*}{34} & \multirow{2}{*}{ Male } & \multirow{2}{*}{ Bilateral } & Abducens Nerve (VI) & No lateral eye movements \\
\hline & & & & Facial Nerve (VII) & Facial palsy \\
\hline \multirow{2}{*}{ MBS3 } & \multirow{2}{*}{37} & \multirow{2}{*}{ Female } & \multirow{2}{*}{ Bilateral } & Abducens Nerve (VI) & No lateral eye movements \\
\hline & & & & Facial Nerve (VII) & Facial palsy \\
\hline \multirow{2}{*}{ MBS4 } & \multirow{2}{*}{25} & \multirow{2}{*}{ Female } & \multirow{2}{*}{ Monolateral sx } & \multirow{2}{*}{ Facial Nerve (VII) } & No lateral eye movements \\
\hline & & & & & Facial palsy \\
\hline MBS5 & 27 & Female & Monolateral sx & Facial Nerve (VII) & Facial palsy \\
\hline \multirow{2}{*}{ MBS6 } & \multirow{2}{*}{22} & \multirow{2}{*}{ Female } & \multirow{2}{*}{ Bilateral } & Abducens Nerve (VI) & No lateral eye movements \\
\hline & & & & Facial Nerve (VII) & Facial palsy \\
\hline \multirow{2}{*}{ MBS7 } & \multirow{2}{*}{53} & \multirow{2}{*}{ Female } & \multirow{2}{*}{ Bilateral } & Abducens Nerve (VI) & No lateral eye movements \\
\hline & & & & Facial Nerve (VII) & Facial palsy \\
\hline \multirow{2}{*}{ MBS8 } & \multirow{2}{*}{62} & \multirow{2}{*}{ Female } & \multirow{2}{*}{ Monolateral dx } & Abducens Nerve (VI) & No lateral eye movements \\
\hline & & & & Facial Nerve (VII) & Facial palsy \\
\hline \multirow{2}{*}{ MBS9 } & \multirow[t]{2}{*}{37} & \multirow{2}{*}{ Male } & \multirow{2}{*}{ Monolateral sx } & Abducens Nerve (VI) & No lateral eye movements \\
\hline & & & & Facial Nerve (VII) & Facial palsy \\
\hline \multirow{2}{*}{ MBS10 } & \multirow{2}{*}{51} & Male & Bilateral & Abducens Nerve (VI) & No lateral eye movements \\
\hline & & 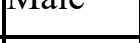 & Dinalual & Facial Nerve (VII) & \\
\hline & 20 & e & & Abducens Nerve (VI) & No lateral eye mover \\
\hline & 20 & e & Dilateral & Facial Nerve (VII) & \\
\hline
\end{tabular}

Table 1. Demographic data and clinical information of MBS participants.

\subsection{Stimuli}

We used the same stimuli as Heberlein and colleagues (2008; for a detailed description of their use in previous studies, see also Vaidya, Jin, \& Fellows, 2013; Adolphs, Jansari, \& Tranel, 2001; Jansari, Tranel, \& Adolphs, 2000). These stimuli are morphs of a single individual's posed emotional faces from the Ekman and Friesen's (1976) stimulus set. The stimuli were selected from 19 linear morphs between a neutral expression and a fully posed emotional expression, for each of six basic emotion categories (i.e. anger, disgust, fear, happiness, sadness, surprise; see Figure 1 for examples). 


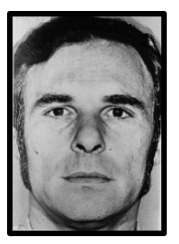

Neutral
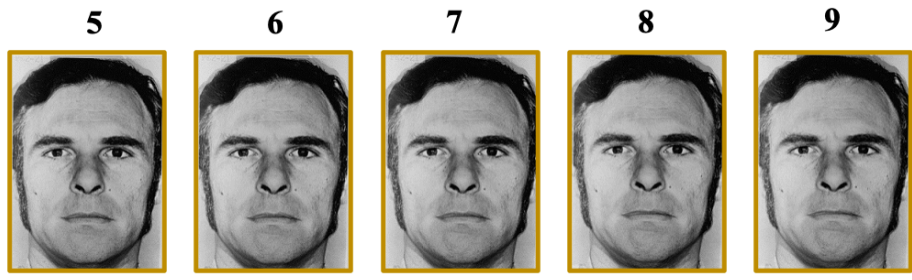

Anger
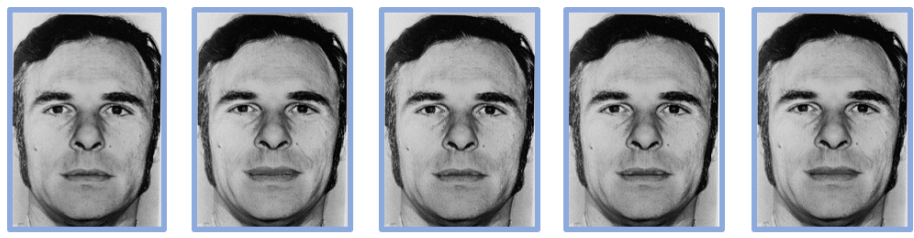

\section{Happiness}

Figure 1. Examples of the morphed face stimuli. The figure depicts a morph at step 5 to 9 of 19 steps between neutral and angry (top), and happy (bottom) facial expressions.

The stimuli were chosen to avoid floor and ceiling effects, based on a sample of normal subjects tested for this purpose (Jansari et al., 2000). Twenty-five faces were shown in total: two afraid, five angry, three disgusted, five happy, five sad, two surprised, and three neutral.

Subjects were presented with one face at a time and rated each in series, using 10-point (1-10) Likert scales. All 25 faces were rated on one emotion, then on the next, and so on for each of the six emotion categories. Thus, the entire stimulus set was shown six times. Emotions were always rated in the same order: fear, happiness, anger, surprise, disgust, and sadness. Consequently, we obtained 6 emotion blocks, each with the name of the specific emotion to be evaluated. No time limit was imposed (see Figure 1 for a schematic representation of the experimental paradigm). All images have been resized to subtend at a visual angle between 10 and 12 degrees. The participants were seated at $70 \mathrm{~cm}$ from the screen. Stimuli were presented using E-Prime on a 13- LCD monitor with $600 \times 800$ of resolution and $75 \mathrm{~Hz}$ of refreshing rate (see Figure 2). 


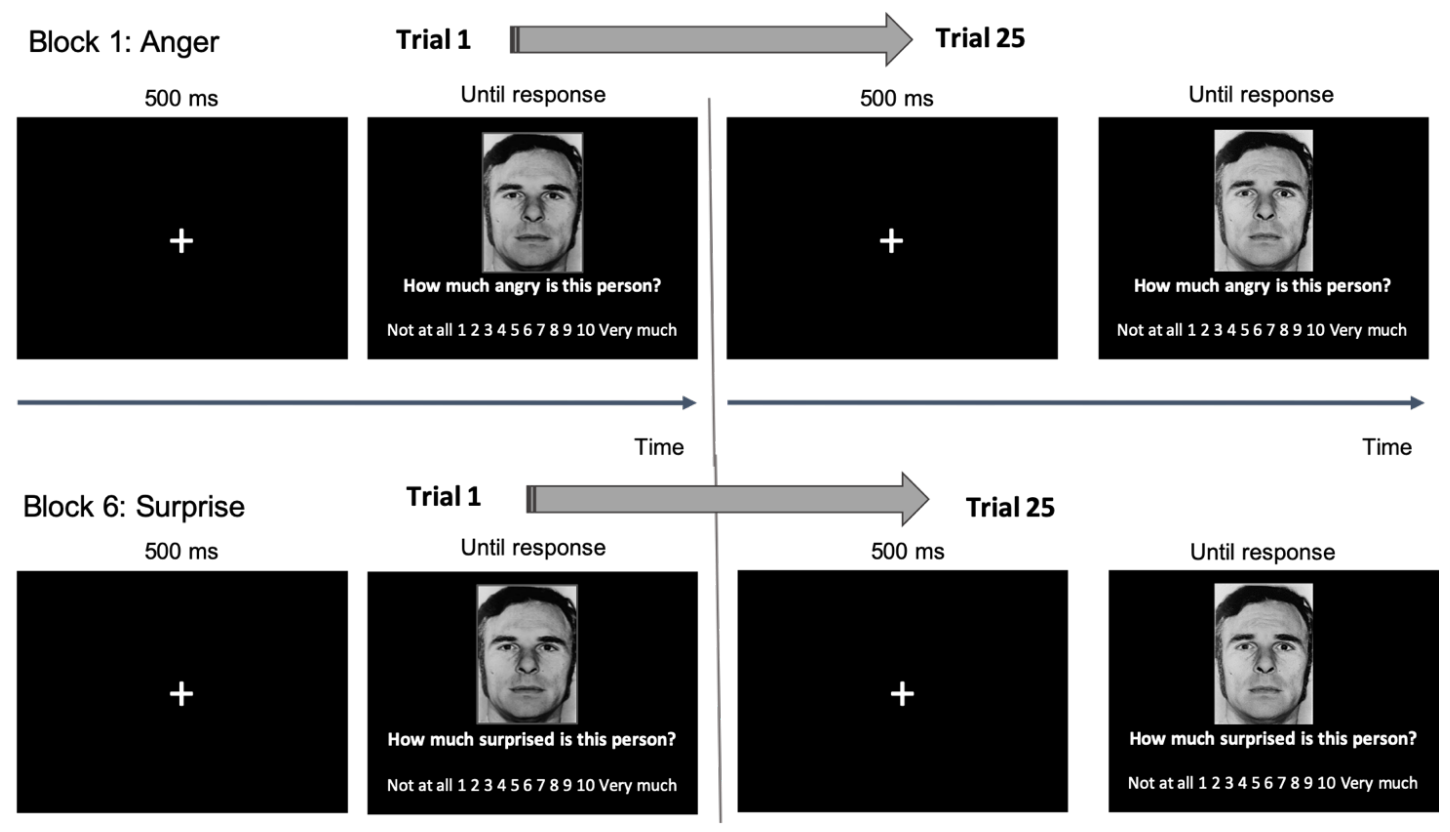

Figure 2. Schematic representation of experimental paradigm.

\subsection{Statistical analysis}

Following Heberlein and colleagues (2008), we used a primary measure of emotion recognition performance. We calculated the difference between ratings given by each subject to each stimulus and that same subject's average rating of the three neutral stimuli within the block. This procedure was intended to correct for any baseline biases in subjects' rating tendencies. The mean difference score was then calculated for each set of predefined emotional faces, collapsing across morph degree. This provided a simple summary measure of the detected intensity of each emotion, in each subset of emotional faces.

To perform statistical comparison of ratings, two different non-parametric tests have been used, namely the Wilcoxon rank sum test, which is carried out when there is no match between compared groups (e.g. comparing the ratings of an emotional stimulus category vs. the rating of the three neutral ones) and the Wilcoxon signed rank test, which is used when the two samples are paired. 
These tests were applied to investigate differences in intensity rating between the two groups and to study the normal phenomenon of the confusability of certain emotions. For example, healthy subjects tend to detect some degree of sadness in the stimulus that experimenter has defined as angry. In this vein, by comparing MBS and control groups' ratings we could evaluate how well emotions are characterized with respect to each other and to the neutral expression.

Furthermore, to compare and evaluate the similarity (or dissimilarity) among the emotional stimuli in the two groups, we performed the Multi-Dimensional Scaling (MDS) by computing the distance among the stimuli according to the different ratings given by the subjects and then computed the best representation of their relative positions on two dimensions (de Leeuw \& Mair, 2009). The stress-value has been used to check the goodness of fit of the MDS (Kruskal, 1964). In addition, we considered the several limitations identified in the use of the stress-value, such as the relation to the sample dimension and the inability to identify whether an observed result is similar or statistically different from a random one. Hence, we tested the goodness of fit of our MDS with the method based on permutations (Mair, Borg, \& Rusch, 2016; Mair, Groener, \& de Leeuw, 2019).

The dataset and analyses reported in this manuscript are available ad Open Science Framework repository: https://github.com/GiulioCaperna/EmotionIntensity

\section{Results}

Perceived intensity of the primary emotion. For primary emotion, we refer to the main emotion that can be perceived by observing the face; thus, we report here to the specific case in which participants were observing an emotional facial expression and were asked to rate the intensity of the correct emotion, e.g. happy face and they had to rate the happiness perceived in that facial expression. Firstly, we examined overall differences in emotional face ratings between the two groups across the six emotions. Thus, we performed comparisons between the relative ratings of the two groups using the Signed-Rank test (see Table 2). 


\begin{tabular}{|c|c|c|c|c|}
\hline Stimulus & Median difference & Confidence Interval & V-Stat & $P$-value \\
\hline Sad & -1.833 & $(-2.50 ;-1,17)$ & 291.5 & $<.001$ \\
\hline Fearful & -3.333 & $(-4.66 ;-1,83)$ & 9.0 & $<.001$ \\
\hline Happy & .000 & $(-.67 ; 1.00)$ & 733.5 & .877 \\
\hline Angry & -.333 & $(-1.33 ; 0.83)$ & 656 & .601 \\
\hline Surprised & -1.000 & $(-3.33 ; 0.67)$ & 84.5 & .178 \\
\hline Disgusted & -1.333 & $(-2.33 ;-0.33)$ & 120 & $<.001$ \\
\hline
\end{tabular}

Table 2. Difference between the rating expressed by the MBS and the control group.

In Table 2 we report the differences in ratings given to the primary emotion according to the two groups. As shown, there are significant differences in the ratings expressed by the MBS with respect to the control group, in particular, for some emotion categories, i.e. sadness, fear and disgust, in which the estimated confidence interval of the median difference is always lower than zero, confirming that the rating expressed by the control group was higher than that expressed by the MBS group. It is important to note that the direction of this trend is present for each facial expression of emotion, except for happiness (in the case of the happiness, the estimated difference between the two groups is exactly zero ${ }^{2}$ ) but reaches significance only for the three emotions reported above.

Sensitivity to the primary emotion. Here, as in the previous paragraph, we report ratings given by the control and MBS group, which reflect the ability to correctly detect an emotion as present when present (primary emotion). Figure 3 depicts the difference in the judgement in all levels of intensity of each facial expression by the two groups. In particular, the results of the difference between the average ratings of the primary emotion (i.e. when participants had to rate the level of a specific emotion when exactly that emotion was presented) and the average ratings of the neutral faces presented in the same block (e.g. the rating expressed by participants to neutral faces in the block 1 was subtracted to the

\footnotetext{
${ }^{2}$ The tests based on the classic paired t-test, give the same three emotions (i.e. sadness, afraid and disgusted) as statistically significant. The interpretation of the signed rank test is somehow similar to the t-test but it is based on the median of difference instead of the difference between means. Having matched observations, the procedure estimates the median of the differences among paired subjects. Consequently, if the median difference between two groups is negative, the second group is intended to have larger values. In this case, the control group expressed significantly higher ratings than MBS group when asked about sad, fearful and disgusted faces.
} 
rating of the images evaluated in the same block). The presence of a dot in Figure 3 indicates that the ratings for that stimulus are statistically different from the ratings of the neutral stimuli in the same block, according to the Rank-Sum test performed with threshold level alpha $=0.05$. Given the intrinsic characteristic of each emotion of being more intense than the neutral one, we used a one-sided test to investigate the difference between groups (significant test values of all comparisons are reported in the Supplemental materials section in Table S1). This result is in line with the previous one shown in Table 2.

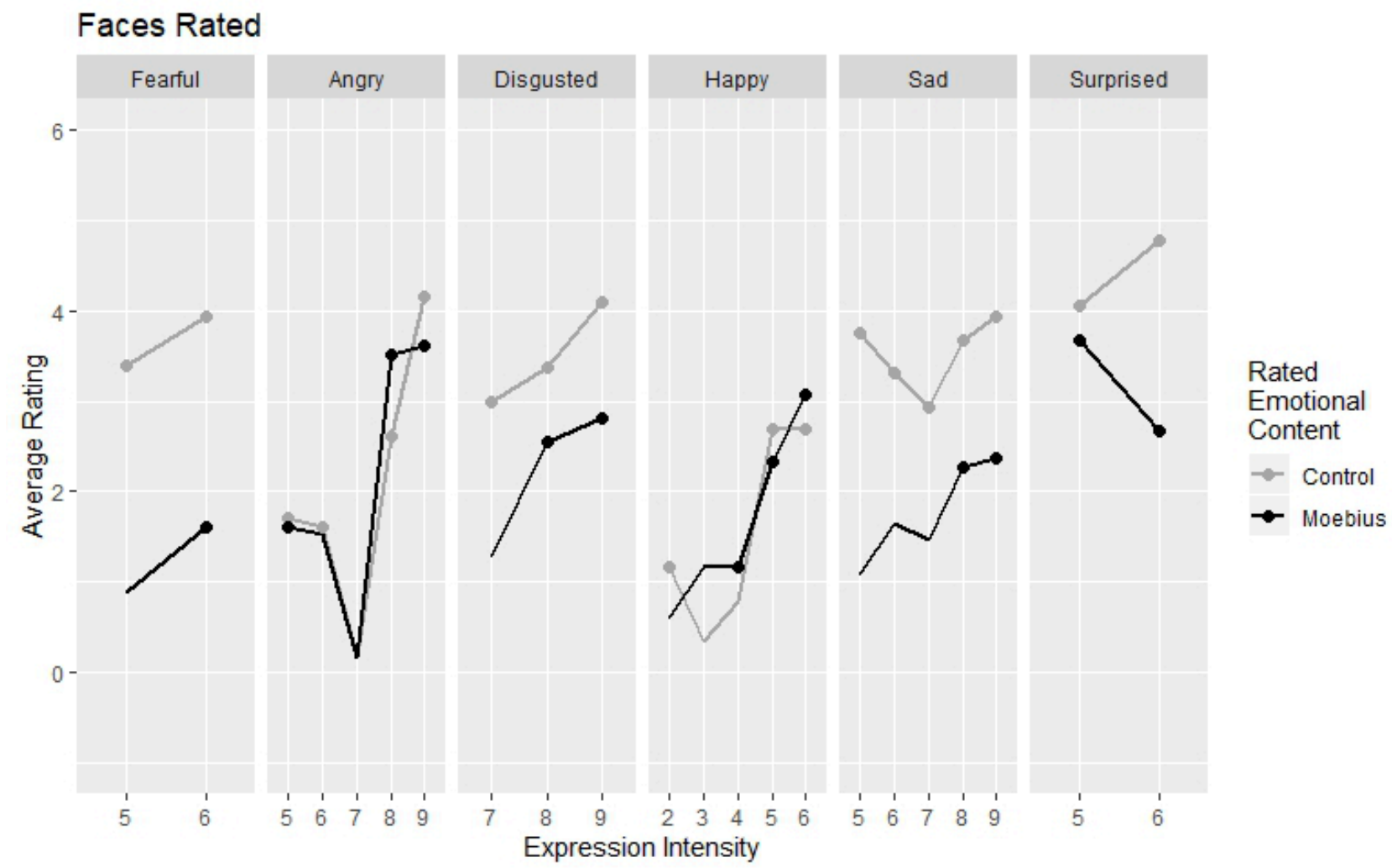

Figure 3. Relative ratings of the primary emotion evaluated in each specific emotion block for the two groups. In this graph, we report the primary emotion only. The name of the column represents the facial expression seen and evaluated by participants. In the graph, the $x$-axis represents morph degree, from most neutral on the left to most intense emotion on the right; the y-axis represents the rating assigned on Likert scales. The values shown in the Figure are relative, because they are the result of the difference between the original rating in the same blocks of those stimuli, and the rating of the neutral stimuli in the same blocks. When a ball is present, it means 
that the ratings expressed for that stimulus are statistically different from the ratings of the neutral stimuli in the same block, according to the Rank-Sum test performed with threshold level alpha=0.05.

To ensure that the difference found in ratings of emotional faces was not driven by differences in baseline ratings, we next compared subjects' ratings of the three neutral faces on each of the six emotions. According to the Signed-rank tests performed to compare the two groups, there is no statistically significant evidence of a difference between the control group and MBS group in the evaluation of the emotional intensity of the neutral stimuli for any emotion block categories $(\min p=$ 0.062; $\mathrm{V}=142$, for happiness block).

Confusability of emotions. Here, we focus on investigating the perception of distinct emotions that people report experiencing when they observe a facial expression. In this vein, it is possible to understand whether other emotions are perceived inside the primary one and to understand participants' ability to discriminate emotions from a neutral facial expression, by taking the ratings given to the neutral as a reference. In Figure 4, we compared the ratings given to the non-present emotions (e.g. when participants were rating the sadness of a stimulus, which had a happy expression instead) to the neutral ones. The presence of a colored dot in Figure 4 indicates that the ratings for that stimulus are statistically different from the ratings of the neutral stimuli in the same block, according to the RankSum test performed with threshold level alpha $=0.05$. Again, given the intrinsic characteristic of each emotion of being more intense than the neutral one, we used a one-sided test to investigate the difference between groups (significant test values of all comparisons are reported in the Supplemental materials section in Table S2). 


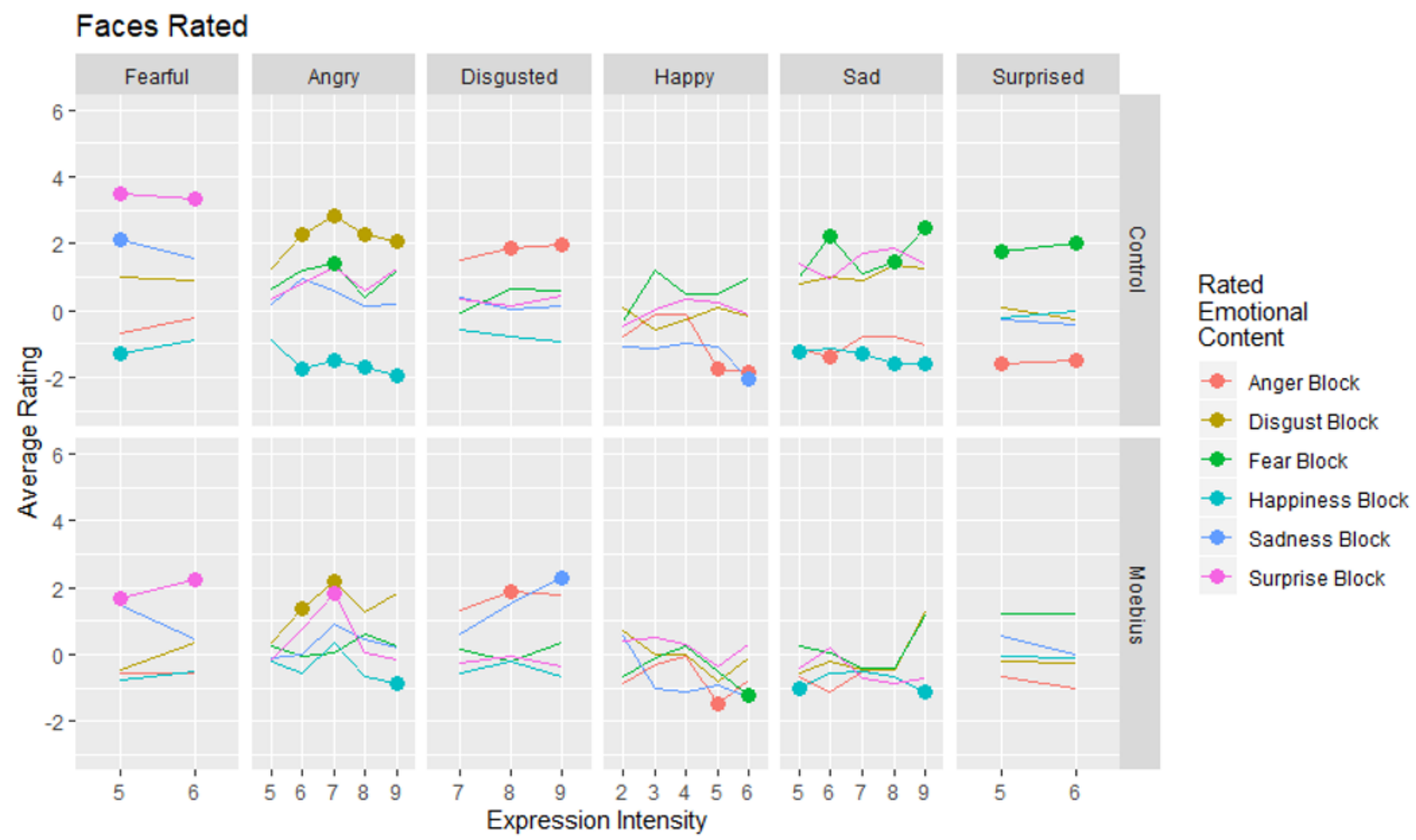

Figure 4. Relative ratings of non-present emotions evaluated in each specific emotion block for the two groups. In this graph, we report all the ratings of each evaluated emotions, excluding the primary one (i.e. the one actually portrayed in the stimulus). The first line of the graph shows the results of the control group, while the second row represents the results of the MBS one. The name of the column represents the facial expression seen by participants, while in the legend the asked emotion. In the graph, the $x$-axis represents morph degree, from most neutral on the left to most intense emotion on the right; the y-axis represents the rating assigned on Likert rating scales. The values shown in the Figure are relative, because they are the result of the difference between the original rating in the same blocks of those stimuli, and the rating of the neutral stimuli in the same blocks. When a colored ball is present, it means that the ratings expressed for that stimulus are statistically different from the ratings of the neutral stimuli in the same block, according to the Rank-Sum test performed with threshold level alpha $=0.05$.

It is possible to observe that the control, compared to the Moebius group, perceived a more considerable amount of other emotions inside the primary ones. For example, the control group, evaluated the angry facial expression, "angry" column, also as disgusted, as suggested by the significant difference for almost all the degrees of disgust, compared to the neutral one. In other words, when they were observing an angry facial expression, they also detected the presence of disgust. On 
the contrary, when they were looking at an angry facial expression, they did not detect any degrees of happiness as suggested by the ratings given to this emotion, which are significantly lower than the neutral one. The same pattern is noticeable for sadness and surprise.

Discriminability between primary and secondary blended emotion. Here, we report the participant's ability to discriminate the primary to the secondary blended emotion. Thus, we investigated the distance between the ratings given by participants when they were evaluating the primary emotion (e.g. when participants evaluated the intensity of sadness of a facial expression, which is actually expressing sadness) with the secondary blended emotion (i.e. the most competitive one, indicating the one which took the highest values, immediately after the first one), within the observed stimulus (Figure 5). For instance, in the top right cell of the graph in Figure 5, the ratings of surprise which is exactly the emotion expressed by the stimuli - are tested against the ratings given in the fear block, which has the second rating, being the highest of the alternatives to the surprise block. In this case, the Signed-Rank test (two-sided) has been performed to check the presence of a difference between the alternatives (Table S3 in the Supplemental Material section lists the comparisons between the relative ratings of the two groups and shows the results of the Signed-Rank test for all comparisons). When a colored ball is present, it means that the ratings expressed for that stimulus are found to be statistically different from the ratings of the neutral stimuli in the same block. 


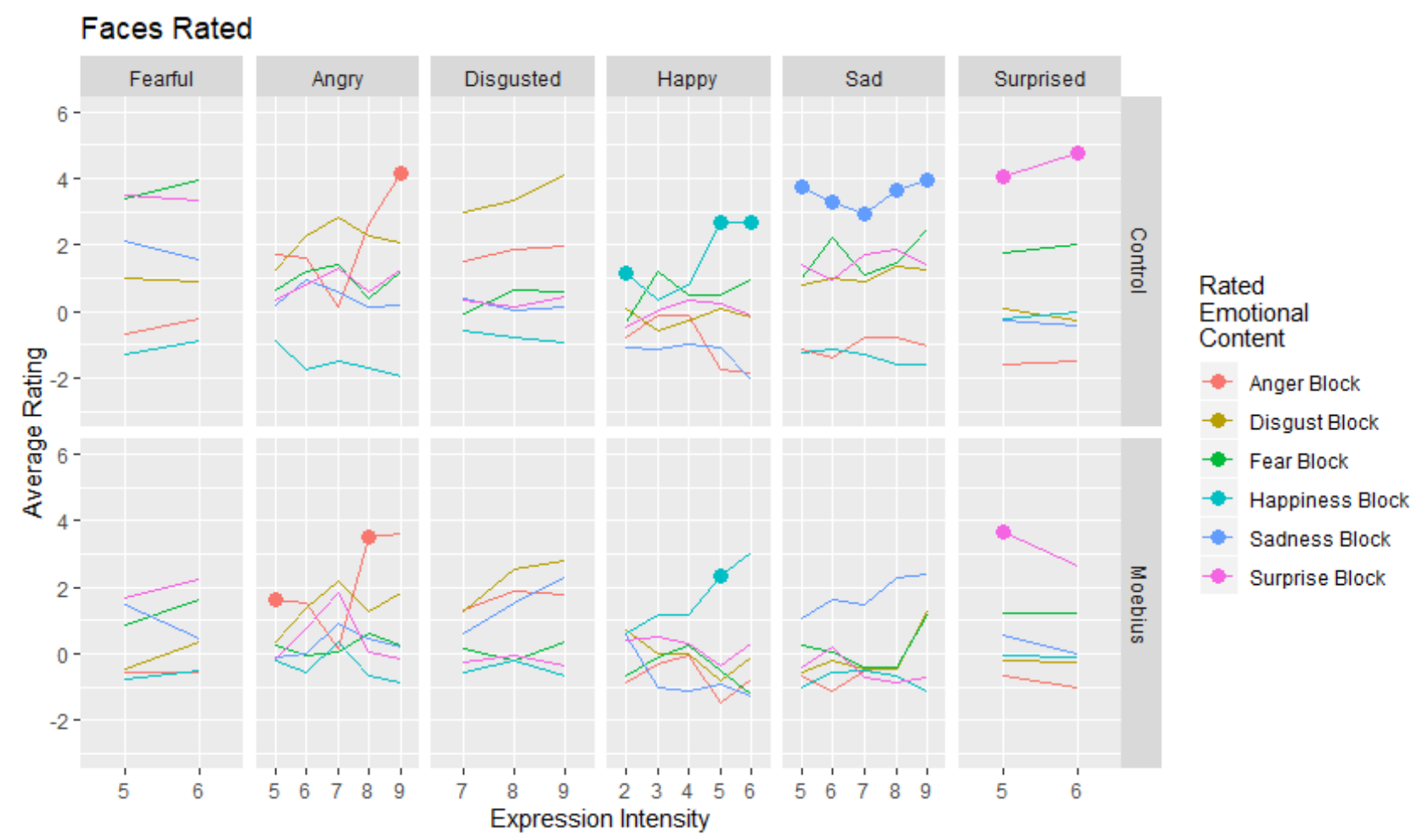

Figure 5. Relative ratings for each emotion stimulus category compared to the secondary blended emotion (i.e. the most competitive one). The first line of the graph shows the results of the control group, while the second row represents the results of the MBS one. The name of the column represents the facial expression seen by participants, while in the legend the asked emotion. In the graph, the $x$-axis represents morph degree, from most neutral on the left to most intense emotion on the right; the y-axis represents the rating assigned on Likert rating scales. The values shown in the Figure are relative, because they are the result of the difference between the original rating in the same blocks of those stimuli, and the rating of the neutral stimuli in the same blocks. When a colored ball is present, it means that the ratings expressed for that stimulus are found to be statistically different from the ratings of the neutral stimuli in the same block, according to the Signed-rank test performed with threshold level alpha $=0.05$.

All the analyses presented above allow to take informed conclusions on the different ability of the two groups in the discrimination of emotions' intensity and is based on direct stimulus-wise comparisons. Nevertheless, we want to describe the relative distance of the stimuli defining a sort of map of the stimuli based on subjects' perception, with a specific focus on the differences we could spot between the two groups. Thus, we computed the maps of the stimuli using the method of MultiDimensional Scaling (MDS) according to the two groups' ratings (control and MBS). Through this 
method it is possible to derive a graphical representation of the relationship among the stimuli (i.e., emotional facial expressions) in terms of distances within a space, such as ratings of perceived similarity (Shepard, 1962; 1980; Takehara \& Suzuki, 2001) and obtain a geometric representation by placing the emotional facial expression stimuli judged as more similar closer together and those emotional facial expressions judged as more dissimilar farther apart. Two solutions were extracted from the multidimensional scaling analysis, one for each group. The number of dimensions extracted is based on the observation of the stress measure on the control group varying the number of dimensions $^{3}$. Namely, $d=1 \mathrm{~S}=0.382, \mathrm{~d}=2 \mathrm{~S}=0.116, \mathrm{~d}=3 \mathrm{~S}=0.65$. These stress-values suggest the use of two dimensions, since the reduction induced by the second dimension is more impactful than the following ones ${ }^{4}$. The two-dimensional solutions are shown in Figure 5. The test on goodness of fit based on the stress measure rejects the null hypothesis of a configuration obtained from a random permutation 5 .

\footnotetext{
${ }^{3}$ Namely, for $\mathrm{d}=1 \mathrm{~S}=0.382, \mathrm{~d}=2 \mathrm{~S}=0.116, \mathrm{~d}=3 \mathrm{~S}=0.065, \mathrm{~d}=4 \mathrm{~S}=0.041$ in the control Group and $\mathrm{d}=1 \mathrm{~S}=0.385$, $\mathrm{d}=2 \mathrm{~S}=0.203, \mathrm{~d}=3 \mathrm{~S}=0.132, \mathrm{~d}=4 \mathrm{~S}=0.09$

${ }^{4}$ We investigated the presence of an "elbow" in the decreasing Stress versus Dimensions function, similar to screen tests in factor analysis, as suggested in Borg, Groener, \& Mair (2018)

${ }^{5}$ For control subjects: $\mathrm{n}=23$, permutations $=1000$, observed stress $=0.116$, $\mathrm{p}$-value $<0.001$; for Moebius subjects: $\mathrm{n}=$ 23 , permutations $=1000$, observed stress $=0.203$, p-value $<0.001$.
} 


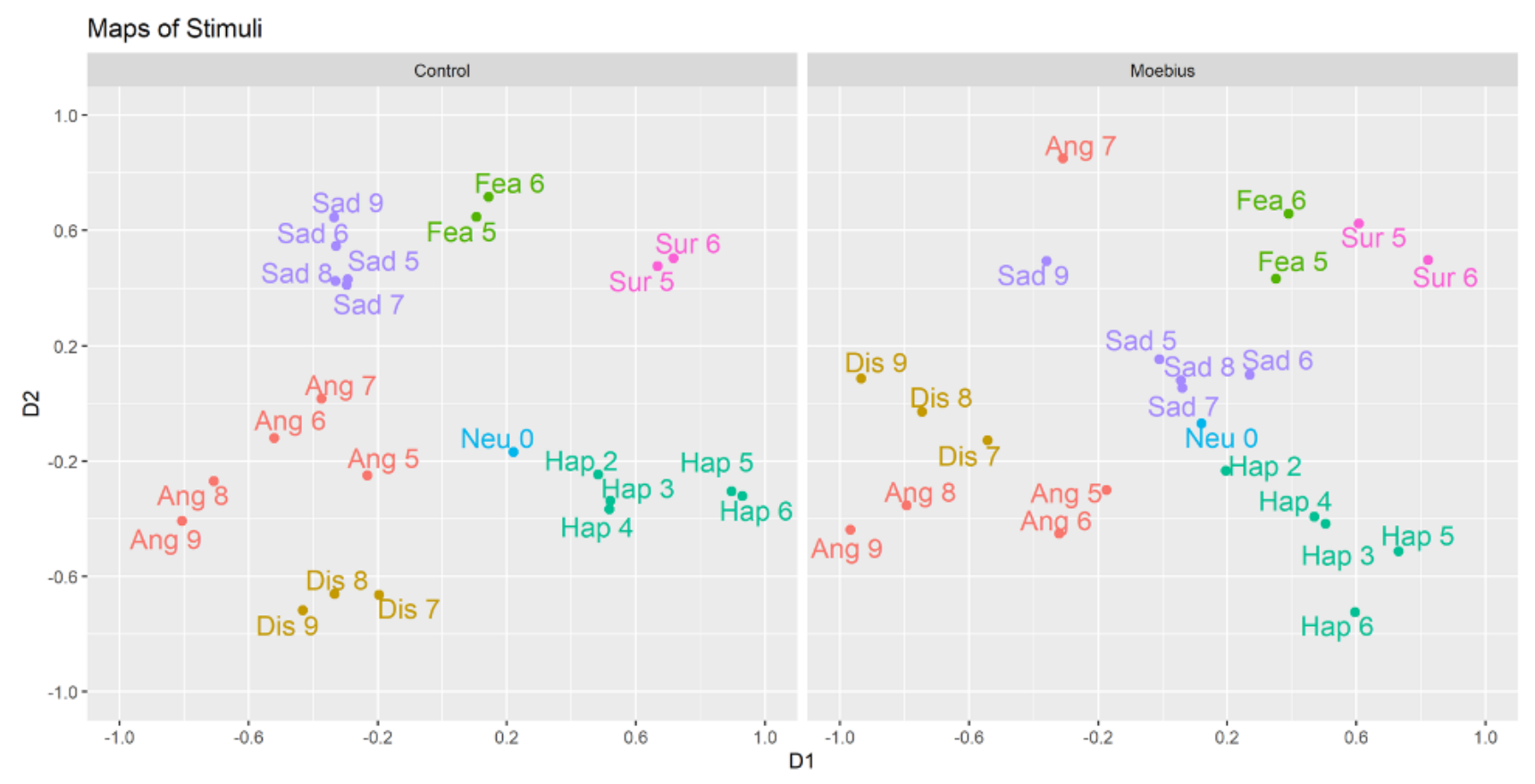

Figure 6. Maps of the 23 stimuli according to the control and MBS group. The coordinates of the dots result from the MDS and represent the best position possible to describe the distance between the stimuli in two dimensions.

In the map of the control group, at the left-hand side, emotions stimuli are sharply grouped; indeed, it is possible to distinguish each emotion category, independently from their intensity level. On the contrary, in the map of the MBS group, the distance between ratings given to different emotion categories is less pronounced, and the intensity of each emotion category plays a more substantial role. At first glance, by comparing the two maps of emotional stimuli, it is noticeable that emotions reach different locations, depending on the group, especially with reference to the neutral emotion. The sad emotion category represents the best example; in the map of the control group, each level of sadness appears tightly grouped to each other and far from the neutral emotion, while, in the map of the MBS group, it appears more spread, and closer to the position of the neutral emotion. This datum is in line with the results reported in Table S1 (see Supplementary Materials) and may suggest that the difference between the two groups is higher for low than high-intensity facial expressions. The same pattern can be observed for other emotions (i.e. fear, surprise, disgust and anger emotions), which appear further 
from the neutral category in control than in the map of MBS. This pattern suggests that the control group seems to use the space of the map better, therefore that each emotion is well defined and characterized by the others.

By taking all the results together, we can notice that some levels are more blended than others; the seventh level of the anger emotion is often misinterpreted by both groups when asked how angry the expression is (see Figure 2). Indeed, both groups confused anger with disgust, but only the MBS interpreted that emotion as surprise (Figure 3). Notably, the seventh level of anger emotion is also the one which makes the main difference in the position on the map (Figure 5).

\section{Discussion}

The present investigation aimed at examining the role of facial mimicry and sensorimotor simulation in processing emotions. It compared the judgements of a group of MBS to a group of healthy participants matched for age and gender. The MBS population represents an optimal model to better understand the role of facial mimicry in emotional processing, given the congenital neurological disorder associated with this syndrome that primarily affects the muscles controlling facial expressions. For this purpose, subjects participated in a sensitive task using faces morphed between neutral and posed emotional expressions, as Heberlein and colleagues (2008). This task allows estimating both the perceived intensity of each level of emotion categories and how well categorized an emotion is in relation to the others, as an index of their confusability. In order to investigate these two aspects, we compared ratings using two different non-parametric tests (i.e. the Wilcoxon rank-sum test and the Wilcoxon signed-rank test), and we also employed the technique of Multi-Dimensional Scaling to produce spatial models, summarizing the similarities perceived among emotions levels of each facial emotion expression category.

As already addressed in the Introduction, MBS subjects show significant variability in emotion recognition deficits, and the results presented in the literature are sometimes ambiguous and not 
conclusive (Giannini, Tamulonis, Giannini, Loiselle, \& Spirtos, 1984; Calder, Keane, Cole, Campbell, \& Young, 2000; Bogart, \& Matsumoto, 2010; Vannuscorps, \& Caramazza, 2020). Such variability could be accounted by several factors. On the one hand, previous studies employed tasks that are not very sensitive. It is possible in fact that deficits in simulation can be more clearly highlighted by the implementation of particularly sensitive tasks (Ferrari \& Sessa, in preparation). On the other hand, it is possible that the visual analysis mechanism should be considered a complementary rather than alternative mechanism to simulation and that subjects with MBS, in the course of their development, have learned to use this complementary mechanism in a more refined way. In the present study we have implemented a particularly sensitive task, which seems more effective in detecting more subtle simulation deficits. In particular, we were interested in both differences in the intensity level perceived by the two groups, and a global measure of 'correctness', an index that is affected both by ratings of the target emotion and by ratings of all five of the other emotion categories. Indeed, by comparing ratings given to facial expressions of emotions to ratings given to the neutral ones, it is possible to track the ability to detect an emotion when present, and to correctly rate it as absent when absent, along the whole continuum.

Overall, we found that the MBS group was impaired at recognizing emotions from facial expressions, as measured by a sensitive test of facial emotion recognition. This deficit was observed in groupwise analyses of mean ratings across different intensities of emotion expression for three specific emotion categories, i.e. sadness, fear and disgust. In the framework of simulation theories, recent models have described how the role of facial mimicry can play a pivotal role not only in recognition of someone else's emotions but also in the evaluation of intensity (Wood et al. 2016; Goldman \& Sripada, 2005). Further, as already mentioned, different studies have found that botulinum toxin-A injection weakened the perception of slightly emotional facial expressions stimuli (Baumesteir, Papa, \& Foroni, 2016; Davis et al., 2011). This result overlaps with other findings in which subtle stimuli 
(e.g. low in emotional intensity) were particularly affected by impairments of facial mimicry (Oberman, Winkielman, \& Ramachandran, 2007).

Notably, our findings also reveal that MBS group do not show facial emotions recognition impairments for other facial expressions, more specifically happiness. This is in line with other studies carried out in children (Herba, Landau, Russell, Ecker, \& Phillips, 2006; Nicolini et al. 2019; De Stefani et al. 2019). Some authors have proposed that facial expression of happiness is easier to recognize (De Stefani et al. 2019; Rosenberg, McDonald, Dethier, Kessels, \& Westbrook, 2014) as it has a unique unambiguous configuration, which implies motor patterns, which are clearly different from all other facial expressions. Thus, configurational features (e.g. corner of the lips directed upwards) may be used to recognize positive facial expressions also when motor simulation mechanisms are altered or partially lesioned. From a developmental perspective, it has been shown that young children are more accurate at recognizing positive emotions (Garcia \& Tully, 2020). This result has also been shown in studies involving adults (Palermo \& Coltheart, 2004; Leppänen \& Hietanen, 2004), in line with the fact that it is the only positive of the basic emotions (Ekman, 1992) and hence should be most distinct.

Such positive bias has been interpreted also as the consequence of early experience where infants and children are normally engaged in positive interactions with a robust exposure to the expression (Bai, Repetti, \& Sperling, 2016). However, there could be also biological explanations for such positive bias since there are different developmental trajectories in the emergence of different facial expressions, supporting specific functions and potentially differentiated mechanisms of fine motor control.

A secondary aim of the present investigation was to evaluate the ability to detect an emotion when present, thus we compared the ratings expressed by each group both for each coherent emotion and for the neutral one. We found that the MBS group, compared to the control group, did not judge some levels of intensity of facial expressions of fear, disgust and sadness as more intense than the 
neutral expression. Importantly, the neutral facial expression intensity was not evaluated differently by the two groups; thus, the difference we found is entirely attributable to the emotion categories, suggesting that lower levels of those emotions were more likely confused with the neutral facial expressions.

As already mentioned, despite there is no agreement in the few studies investigating facial expression recognition ability in MBS subjects (Calder et al. 2000; Bate, Cook, Mole, \& Cole, 2013), some studies revealed some significant difficulties in this ability, as a consequence of the facial motor deficits. Notably, few studies (De Stefani et al., 2019; Nicolini et al., 2019), having found a weaker temperature change in MBS children's autonomic response to emotional stimuli compared to the control group, proposed that the impairment of facial movements attenuates the intensity of the emotional experience. It is therefore possible that the reduction in the experienced intensity of an emotion attenuated also the intensity judgments attributed to the emotion conveyed by a facial expression. It is worth noting, that subjects suffering from other clinical conditions, involving the capacity to control face movements (e.g. Parkinson and Huntington's disease) also reveal clear impairments in facial expression recognition performance. These studies found impairments both in the imitation of emotional facial expressions and in the emotion recognition processes (Ricciardi, Comandini, \& Erro, 2017; Trinkler et al. 2017; Gray \& Tickle-Degnen, 2010; Sprengelmeyer et al., 1996), probably linked to the reduced facial musculature and their consequent inability to simulate emotional expressions (Marneweck et al., 2014). Our results support the conclusions of previous studies investigating other clinical conditions involving facial motor deficits, and, by leveraging the MBS unique condition, it further supports the idea that even in the absence of central motor deficits (i.e. cortical or subcortical), peripheral facial motor nerve deficiency impacts on the mechanisms involved on facial expression recognition. Current findings are in agreement with motor simulation theories, which assert that in order to evaluate the type and intensity of a facial expression, the ability to simulate the same expression must be preserved. 
Furthermore, by comparing ratings between the non-present emotion (e.g. the rating given to a happy facial expression when asked participants to evaluate sadness) and the neutral one, we could obtain a measure of the present amount of each asked emotion within the one seen by participants, taking the neutral as a reference.

In this vein, if we look at the ratings expressed by participants, it is also possible to know both which emotions are perceived in each emotion category because the ratings are higher than the one expressed for the neutral, and also which emotions are not perceived within each category because the ratings are lower than the one expressed for the neutral (Figure 4). For example, by looking at the rating expressed by the control group when these participants observed an angry facial expression, it is possible to trace the presence not only of the primary emotion, i.e. anger, but also of other emotions which are contained in the primary one, since they were rated as more intense than neutral facial expressions. In this case, when the control group were looking at an angry facial expression, they detected some degree of disgust in the experimenter-defined angry stimuli. However, they did not detect any degree of happiness, as expressed by the rating significantly lower than the one given to the neutral stimuli, when they were looking at an angry face. Conversely, the MBS group did not perceive any other emotions when looked at an angry facial expression, as suggested by the non-significant difference between the ratings given to the emotion and neutral facial expression - except for a few degrees of disgust (Figure 3).

By also taking the Multi-Dimensional Scaling's results into account, it is possible to notice that each emotion categories are sharply grouped in the controls, indicating that, although they may perceive "multiple simultaneous facial expressions" (Richmond \& Croskey, 1999), they have a clear distinction of each emotion category, independently from their intensity level. This result is in line with other studies in which a perceived similarity between some categories of emotions expressed by facial expressions was found in healthy participants (Wegrzyn, Vogt, Kireclioglu Schneider, \& Kissler, 2017; Palermo and Coltheart, 2004). On the contrary, the MBS seemed to give the same rating to the emotions 
and the neutral facial expressions, suggesting that all the emotions categories were less discriminated from the neutral facial expression. Furthermore, this result was confirmed by a less pronounced distance between ratings given to different emotion categories, as shown in Figure 6. Interestingly, our data showed (Figure 5) that disgust, and anger emotions are perceived as blended, both in control and in MBS groups (Ekman \& Friesen, 1998; see also Russell's circumplex model, 1980). Despite this similarity, the proximal location of anger and disgust in the two-dimensional models is higher in the MBS than in the control group, suggesting that these emotions can be easily confused by the MBS. Together these data indicate that the preserved facial musculature confer an advantage over the MBS group by increasing the ability to perceive multiple facial configuration possibilities, and to a better discrimination of the expression. The possibility to use motor simulation during the observation of facial expression seems therefore to be a key mechanism involved in recognizing emotions.

Notably, the happy facial expression is always well interpreted by both groups if compared to the neutral one, suggesting that they were able to detect happiness when present, as suggested by the relative line for the target emotion ratings depicted in Figure 2. However, it should be considered that previous studies have found similar ceiling effects for happy expressions, by using different tasks (e.g., Vicari et al., 2000). This effect found for happiness could be explained by the fact that happiness was the only positive emotion presented and that the facial configuration for happiness is more distinctive than those of other facial expressions (Vicari et al., 2000). In addition to this, the happy expression is the most common and frequently used by parents and others during the course of development, thus providing a variety of contexts in which the child can learn to discriminate it from other expressions without significant cognitive efforts. This interesting result suggests that there may be multiple mechanisms at stake during the visual processing of facial expression. When a facial expression has an unambiguous configuration, such as happiness, the motor simulation might be less relevant in the recognition process, or its involvement is less demanding. In fact, in line with this, it cannot be excluded that a minimal activity of sensorimotor activation is still present in MBS, given that the motor centers 
at cortical level are still preserved and that some residual facial motor activity is still present. Clearly, more accurate neurophysiological investigations should explore such issue.

With regard to the secondary blended emotions, our results suggest that the MBS were less able to discriminate from some levels of the secondary blended emotion, i.e. surprise. The same pattern, even more robust, can be noticed by looking at the ratings given for sad facial expressions; the MBS showed less ability both in detecting this emotion when present (Figure 3) and in discriminating it from the most competitive one, i.e. fear (Figure 5).

In summary, our study provides evidence of a deficit in recognizing emotions in MBS subjects, expressed by a significant decrease in the rating of the intensity of three specific emotion categories, namely sadness, fear and disgust (although the trend was similar for all emotions). Furthermore, we observed an impairment in detecting these emotions, resulting in a stronger confusability of such emotions with the neutral and the secondary, as indicated by the proximity depicted in the MultiDimensional Scaling map. These findings provide support for embodied theories, which hypothesize that sensorimotor systems are involved in the detection and discrimination of emotions (e.g., Niedenthal, 2007). Accordingly, sensorimotor simulation contributes to the subjective experience of others' emotions, so a reduced/altered sensorimotor activity could determine a lower intensity of the emotional experience and, consequently, an altered perception of the intensity of the emotions conveyed by the facial expressions of others. 


\section{References}

Abramson, D. L., Cohen, M., \& Mulliken, J. (1998). Möbius Syndrome: Classification and Grading System. Surgery, Plastic and Reconstructive, 4, 961-967.

Adolph, R. (2002). Recognizing Emotion From Facial Expressions: Psychological and Neurological Mechanisms Ralph. Behavioral and Cognitive Neuroscience Reviews, 1, 21-62.

Adolphs, R, Jansari, A., \& Tranel, D. (2001). Hemispheric perception of emotional valence from facial expressions. Neuropsychology, 516-526.

Adolphs, Ralph. (2009). The Social Brain: Neural Basis of Social Knowledge Ralph. Annual Review of Psychology, 60, 693-716. https://doi.org/10.1146/annurev.psych.60.110707.163514.

Bai, S., Repetti, R. L., \& Sperling, J. B. (2016). Children's expressions of positive emotion are sustained by smiling, touching, and playing with parents and siblings: A naturalistic observational study of family life. Developmental psychology, 52(1), 88.

Bate, S., Cook, S., Mole, J., \& Cole; J. (2013). First Report of Generalized Face Processing Difficulties in Möbius Sequence. Current Science, 8(4), 1435-1439. https://doi.org/10.1371/Citation

Baumeister, J. C., Papa, G., \& Foroni, F. (2016). Deeper than skin deep - The effect of botulinum toxinA on emotion processing. Toxicon, 118, 86-90. https://doi.org/10.1016/j.toxicon.2016.04.044

Bianchi, B., Copelli, C., Ferrari, S., Ferri, A., \& Sesenna, E. (2010). Facial animation in patients with Moebius and Moebius-like syndromes. International Journal of Oral and Maxillofacial Surgery, 39(11), 1066-1073. https://doi.org/10.1016/j.ijom.2010.06.020

Bogart, K. R., \& Matsumoto, D. (2010). Facial mimicry is not necessary to recognize emotion: Facial expression recognition by people with Moebius syndrome. Social Neuroscience, 5(2), 241-251. https://doi.org/10.1080/17470910903395692

Borg, I., Groenen, P. J., \& Mair, P. (2018). Applied Multidimensional Scaling and Unfolding. Springer. Briegel, W. (2006). Neuropsychiatric findings of Möbius sequence -- a review. Clinical Genetics, 
70(2), 91-97. https://doi.org/10.1111/j.1399-0004.2006.00649.

Calder, A. J., Keane, J., Cole, J., Campbell, R., \& Young, A. W. (2000). Facial expression recognition by people with Möbius syndrome. Cognitive Neuropsychology, 17(1-3), 73-87. https://doi.org/10.1080/026432900380490.

Cattaneo, L., Chierici, E., Bianchi, B., Sesenna, E., \& Pavesi, G. (2006). The localization of facial motor impairment in sporadic Möbius syndrome. Neurology, 66(12), 1907 LP - 1912. https://doi.org/10.1212/01.wnl.0000219766.96499.6c.

de la Rosa, S., Fademrecht, L., Bülthoff, H. H., Giese, M. A., \& Curio, C. (2018). Two Ways to Facial Expression Recognition? Motor and Visual Information Have Different Effects on Facial Expression Recognition. Psychological Science, 29(8), 1257-1269. https://doi.org/10.1177/0956797618765477.

de Leeuw, J., \& Mair, P. (2009). Gifi methods for optimal scaling in R: The package homals. Journal of Statistical Software, 31(4), 1-21. https://doi.org/10.18637/jss.v031.i04.

De Stefani, E., Ardizzi, M., Nicolini, Y. et al. Children with facial paralysis due to Moebius syndrome exhibit reduced autonomic modulation during emotion processing. Journal of Neurodevelop Disord 11, 12 (2019). https://doi.org/10.1186/s11689-019-9272-2.

Ekman P. Are there basic emotions? Psychol Rev. 1992;(99):550-553.

Ekman, P., \& Friesen, W. (1976). No Title Pictures of facial affect.

Ekman, P., \& Friesen, W. (1982). Felt false and miserable smiles [Ekman \& Friesen, 1981].pdf. Journal of Nonverbal Behavior, 6(4), 238-252.

Elfenbein, H. A. (2007). Emotion in Organizations: A Review in Stages. Academy of Management, $1(1), 315-386$.

Ferrari, P., Tramacere, A., Simpson, E., \& Iriki, A. (2013). Mirror neurons through the lens of epigenetics. Trends in Cognitive Sciences, 17(9), 450-457. 
Finzi, E., \& Rosenthal, N. E. (2014). Treatment of depression with onabotulinumtoxinA: a randomized, double-blind, placebo controlled trial. Journal of psychiatric research, 52, 1-6.

Fletcher, P. C., Happé, F., Frith, U., Baker, S. C., Dolan, R. J., Frackowiak, R. S. J., \& Frith, C. D. (1995). Other minds in the brain: a functional imaging study of "theory of mind" in story comprehension. Cognition, 57(2), 109-128. https://doi.org/https://doi.org/10.1016/00100277(95)00692-R

Frith, C., \& Frith, U. (2005). Theory of mind. Current Biology: CB, 15(17), 644-645. https://doi.org/10.1016/j.cub.2005.08.041.

Gallese, V. (2016). Embodied simulation as a second-person perspective on intersubjectivity. Neuroscience and Critique: Exploring the Limits of the Neurological Turn, 188-202. https://doi.org/10.4324/9781315714189.

Gallese, V., \& Goldman, A. (1998). Mirror neurons and the mind-reading. Trends in Cognitive Sciences, 2(12), 493-501. https://doi.org/10.1016/S1364-6613(98)01262-5

Gambarota, F., \& Sessa, P. (2019). Visual working memory for faces and facial expressions as a useful "tool" for understanding social and affective cognition. Frontiers in Psychology, 10(OCT), 1-7. https://doi.org/10.3389/fpsyg.2019.02392

Garcia, S. E., \& Tully, E. C. (2020). Children's recognition of happy, sad, and angry facial expressions across emotive intensities. Journal of experimental child psychology, 197, 104881.

Giannini, A., Tamulonis, D., Metthew, M., Giannini, J., Loiselle, R., \& Spiritos, G. (1984). Single case study. Defective Response to social cues in Möbius Syndrome. The Journal of Nervous and Mental Disease, 172(3), 174-175.

Goldman, A. I., \& Sripada, C. S. (2005). Simulationist models of face-based emotion recognition. Cognition, 94(3), 193-213. https://doi.org/10.1016/j.cognition.2004.01.005

Gopnik, A., \& Wellman, H. M. (1992). Why the Child's Theory of Mind Really Is a Theory. Mind \&amp; Language, 7(1-2), 145-171. https://doi.org/10.1111/j.1468-0017.1992.tb00202.x 
Gray, H. M., \& Tickle-Degnen, L. (2010). A meta-analysis of performance on emotion recognition tasks in Parkinson's disease. Neuropsychology, 24(2), 176.

Heberlein, A., Padon, A., Gillihan, S., Farah, M., \& Fellows, L. (2008). Ventromedial Frontal Lobe Plays a Critical Role in Facial Emotion Recognition. Journal of Cognitive Neuroscience, 20, 721733. https://doi.org/10.1162/jocn.2008.20049.

Herba, C. M., Landau, S., Russell, T., Ecker, C., \& Phillips, M. L. (2006). The development of emotionprocessing in children: Effects of age, emotion, and intensity. Journal of Child Psychology and Psychiatry, 47(11), 1098-1106.

Hess, U., \& Blairy, S. (2001). Facial mimicry and emotional contagion to dynamic emotional facial expressions and their influence on decoding accuracy. International Journal of Psychophysiology, 40, 129-141. https://doi.org/10.1016/S0167-8760(00)00161-6

Ipser, A., \& Cook, R. (2016). Inducing a concurrent motor load reduces categorization precision for facial expressions. Journal of Experimental Psychology. Human Perception and Performance, 42(5), 706-718. https://doi.org/10.1037/xhp0000177

Jansari, A., Tranel, D., \& Adolphs, R. (2000). A valence-specific lateral bias for discriminating emotional facial expressions in free field. Cognition and Emotion, 14(3), 341-353. https://doi.org/10.1080/026999300378860

Korb, S., Wood, A., Banks, C. A., Agoulnik, D., Hadlock, T. A., \& Niedenthal, P. M. (2016). Asymmetry of facial mimicry and emotion perception in patients with unilateral facial paralysis. JAMA Facial Plastic Surgery, 18(3), 222-227. https://doi.org/10.1001/jamafacial.2015.2347

Kruskal, J. (1964). Multidimensional Scaling by Optimizing Goodness of Fit a Nonmetric Hypothesis. Psychometrika, 29(1). https://doi.org/10.5137/1019-5149. JTN.14036-15.1

Leslie, A. M. (1994). Pretending and believing: issues in the theory of ToMM. Cognition, 50(1-3), 211-238. https://doi.org/10.1016/0010-0277(94)90029-9

Mair, P., Borg, I., \& Rusch, T. (2016). Goodness-of-Fit Assessment in Multidimensional Scaling and 
Unfolding. Multivariate Behavioral Research, 51(6), 772-789. https://doi.org/10.1080/00273171.2016.1235966

Mair, P., Groenen, P. J., \& de Leeuw, J. (2019). More on multidimensional scaling and unfolding in R: smacof version 2. J. Stat. Softw.[Epub ahead of print].

Marneweck, M., \& Hammond, G. (2014). Discriminating facial expressions of emotion and its link with perceiving visual form in Parkinson's disease. Journal of the Neurological Sciences, 346(12), 149-155.

McIntosh, D. N., Reichmann-Decker, A., Winkielman, P., \& Wilbarger, J. L. (2006). When the social mirror breaks: deficits in automatic, but not voluntary, mimicry of emotional facial expressions in autism. Developmental Science, 9(3), 295-302. https://doi.org/10.1111/j.14677687.2006.00492.x

McKone, E., \& Robbins, R. (2011). Are faces special? In G. Rhodes, A. Calder, M. Johnson, \& J. V. Haxby (Eds.), Oxford Handbook of Face Perception. https://doi.org/10.1093/oxfordhb/9780199559053.013.0009

Montgomery, K. J., \& Haxby, J. V. (2008). Mirror neuron system differentially activated by facial expressions and social hand gestures: A functional magnetic resonance imaging study. Journal of Cognitive Neuroscience, 20(10), 1866-1877. https://doi.org/10.1162/jocn.2008.20127

Nicolini, Y., Manini, B., De Stefani, E., Coudé, G., Cardone, D., Barbot, A., ... Ferrari, P. F. (2019). Autonomic responses to emotional stimuli in children affected by facial palsy: The case of Moebius syndrome. Neural Plasticity, Vol. 2019. https://doi.org/10.1155/2019/7253768

Niedenthal, P. M., Mermillod, M., Maringer, M., \& Hess, U. (2010). The Simulation of Smiles (SIMS) model: Embodied simulation and the meaning of facial expression. Behavioral and Brain Sciences, 33(6), 417-433. https://doi.org/10.1017/S0140525X10000865

Oberman, L. M., Winkielman, P., \& Ramachandran, V. S. (2007). Face to face: Blocking facial mimicry can selectively impair recognition of emotional expressions. Social neuroscience, 2(3- 


\section{4), 167-178.}

Orlowska, A. B., Krumhuber, E. G., Rychlowska, M., \& Szarota, P. (2018). Dynamics Matter: Recognition of Reward, Affiliative, and Dominance Smiles From Dynamic vs. Static Displays. Frontiers in Psychology, 9, 938. https://doi.org/10.3389/fpsyg.2018.00938

Palermo, R., \& Coltheart, M. (2004). SpringerLink - Behavior Research Methods, Volume 36, Number 4. Behavior Research Methods, 36(4), 634-638. https://doi.org/10.3758/BF03206544

Paracampo, R., Pirruccio, M., Costa, M., Borgomaneri, S., \& Avenanti, A. (2018). Visual, sensorimotor and cognitive routes to understanding others' enjoyment: An individual differences rTMS approach to empathic accuracy. Neuropsychologia, 116, 86-98. https://doi.org/https://doi.org/10.1016/j.neuropsychologia.2018.01.043

Paracampo, R., Tidoni, E., Borgomaneri, S., Di Pellegrino, G., \& Avenanti, A. (2017). Sensorimotor Network Crucial for Inferring Amusement from Smiles. Cerebral Cortex, 27(11), 5116-5129. https://doi.org/10.1093/cercor/bhw294

Picciolini O, Porro M, Cattaneo E, Castelletti S, Masera G, Mosca F, et al. Moebius syndrome: clinical features, diagnosis, management and early intervention. Ital J Pediatr. 2016;42:56.

Pitcher, D., Garrido, L., Walsh, V., \& Duchaine, B. C. (2008). Transcranial Magnetic Stimulation Disrupts the Perception and Embodiment of Facial Expressions. Journal of Neuroscience, 28(36), 8929-8933. https://doi.org/10.1523/JNEUROSCI.1450-08.2008

Plutchik, R. (1997). The circumplex as a general model of the structure of emotions and personality. In Circumplex models of personality and emotions. (pp. 17-45). https://doi.org/10.1037/10261001

Pochedly, J. T., Widen, S. C., \& Russell, J. A. (2012). What emotion does the "facial expression of disgust" express? Emotion, 12(6), 1315-1319. https://doi.org/10.1037/a0027998

Ponari, M., Conson, M., D’Amico, N. P., Grossi, D., \& Trojano, L. (2012). Mapping correspondence between facial mimicry and emotion recognition in healthy subjects. Emotion (Washington, D.C.), 
12(6), 1398-1403. https://doi.org/10.1037/a0028588

Pourtois, G., Sander, D., Andres, M., Grandjean, D., Reveret, L., Olivier, E., \& Vuilleumier, P. (2004). Dissociable roles of the human somatosensory and superior temporal cortices for processing social face signals. European Journal of Neuroscience, 20(12), 3507-3515. https://doi.org/10.1111/j.1460-9568.2004.03794.x

Preston, S. D., \& de Waal, F. B. M. (2002). Empathy: Its ultimate and proximate bases. Behavioral and Brain Sciences, 25(1), 1-20. https://doi.org/10.1017/S0140525X02000018

Rasmussen, L., Rian; O, Korshoej, A., \& Christensen, S. (2015). Fatal Complications during Anaesthesia in Moebius Syndrome: A Case Report and Brief Discussion of Relevant Precautions and Preoperative Assessments. International Journal of Anesthesiology \& Research, 3, 116-118. https://doi.org/10.19070/2332-2780-1500030

Ricciardi, L., Visco-Comandini, F., Erro, R., Morgante, F., Bologna, M., Fasano, A., ... \& Kilner, J. (2017). Facial emotion recognition and expression in Parkinson's disease: an emotional mirror mechanism?. PLoS One, 12(1), e0169110.

Richmond, V. P., Croskey, J. C.: Non Verbal Behavior in Interpersonal relations. Allyn \& Bacon Inc. (1999)

Rives Bogart, K., \& Matsumoto, D. (2010). Facial mimicry is not necessary to recognize emotion: Facial expression recognition by people with Moebius syndrome. Social Neuroscience, 5(2), 241251. https://doi.org/10.1080/17470910903395692

Rosenberg, H., McDonald, S., Dethier, M., Kessels, R. P., \& Westbrook, R. F. (2014). Facial emotion recognition deficits following moderate-severe traumatic brain injury (TBI): Re-examining the valence effect and the role of emotion intensity. Journal of the International Neuropsychological Society: JINS, 20(10), 994.

Ruba, A. L., Meltzoff, A. N., \& Repacholi, B. M. (2019). How do you feel? Preverbal infants match negative emotions to events. Developmental psychology, 55(6), 1138. 
Rychlowska, M., Cañadas, E., Wood, A., Krumhuber, E. G., Fischer, A., \& Niedenthal, P. M. (2014). Blocking mimicry makes true and false smiles look the same. PLoS ONE, 9(3), 1-8. https://doi.org/10.1371/journal.pone.0090876

Schiano Lomoriello, A., Maffei, A., Brigadoi, S., \& Sessa, P. (2019). Altering sensorimotor simulation impacts early stages of facial expression processing depending on individual differences in alexithymic traits. Retrieved from https://arxiv.org/abs/1906.06424

Schyns, P. G., Bonnar, L., \& Gosselin, F. (2002). Show Me the Features! Understanding Recognition From the Use of Visual Information. Psychological Science, 13(5), 402-409. https://doi.org/10.1111/1467-9280.00472

Shepard, R. N. (1962) The analysis of proximities: Multidimensional scaling with an unknown distance function. Psychometrika, 27, 125-139, 219-246.

Shepard, R. N. (1980). Multidimensional scaling, tree-fitting, and clustering. Science, 210(4468), 390398.

Sessa, P., Schiano Lomoriello, A., \& Luria, R. (2018). Neural measures of the causal role of observers' facial mimicry on visual working memory for facial expressions. Social Cognitive and Affective Neuroscience, 13(12), 1281-1291. https://doi.org/10.1093/scan/nsy095

Sprengelmeyer, R., Young, A. W., Calder, A. J., Karnat, A., Lange, H., Hömberg, V., ... \& Rowland, D. (1996). Loss of disgust: Perception of faces and emotions in Huntington's disease. Brain, 119(5), 1647-1665.

Takehara, T., \& Suzuki, N. (2001). Robustness of the two-dimensional structure of recognition of facial expression: evidence under different intensities of emotionality. Perceptual and Motor Skills, 93(3), 739-753.

Terzis JK, Noah EM. Dynamic restoration in Möbius and Möbius-like patients. Plast Reconstr Surg. $2003 ; 111: 40-55$.

Tramacere, A., Pievani, T., \& Ferrari, P. F. (2017). Mirror neurons in the tree of life: mosaic evolution, 
plasticity and exaptation of sensorimotor matching responses. Biological Reviews, Vol. 92, pp. 1819-1841. https://doi.org/10.1111/brv.12310

Trinkler, I., Devignevielle, S., Achaibou, A., Ligneul, R. V., Brugières, P., de Langavant, L. C., ... \& Bachoud-Lévi, A. C. (2017). Embodied emotion impairment in Huntington's Disease. Cortex, 92, 44-56.

Vaidya, A. R., Jin, C., \& Fellows, L. K. (2014). Eye spy: The predictive value of fixation patterns in detecting subtle and extreme emotions from faces. Cognition, 133(2), 443-456.

Vannuscorps, G., Andres, M., \& Caramazza, A. (2020). Efficient recognition of facial expressions does not require motor simulation. Elife, 9, e54687.

VanSwearingen, J. M., Cohn, J. F., \& Bajaj-Luthra, A. (1999). Specific impairment of smiling increases the severity of depressive symptoms in patients with facial neuromuscular disorders. Aesthetic plastic surgery, 23(6), 416-423.

Vicari, S., Reilly, J. S., Pasqualetti, P., Vizzotto, A., \& Caltagirone, C. (2000). Recognition of facial expressions of emotions in school-age children: the intersection of perceptual and semantic categories. Acta Paediatrica, 89(7), 836-845.

Wegrzyn M, Vogt M, Kireclioglu B, Schneider J, Kissler J (2017). Mapping the emotional face. How individual face parts contribute to successful emotion recognition. PLOS ONE 12(5): e0177239. https://doi.org/10.1371/journal.pone.0177239.

Wingenbach, T. S. H., Brosnan, M., Pfaltz, M. C., Plichta, M. M., \& Ashwin, C. (2018). Incongruence between observers' and observed facial muscle activation reduces recognition of emotional facial expressions from video stimuli. Frontiers in Psychology, 9(JUN). https://doi.org/10.3389/fpsyg.2018.00864

Wolpert, D. M., \& Flanagan, J. R. (2001). Motor prediction. Current Biology: CB, 11(18), 729-732. https://doi.org/10.1016/s0960-9822(01)00432-8

Wood, A., Lupyan, G., Sherrin, S., \& Niedenthal, P. (2016). Altering sensorimotor feedback disrupts 
visual discrimination of facial expressions. Psychonomic Bulletin and Review, 23(4), 1150-1156. https://doi.org/10.3758/s13423-015-0974-5

Wood, A., Rychlowska, M., Korb, S., \& Niedenthal, P. (2016). Fashioning the Face: Sensorimotor Simulation Contributes to Facial Expression Recognition. Trends in Cognitive Sciences, 20(3), 227-240. https://doi.org/10.1016/j.tics.2015.12.010 


\section{CRediT authorship contribution statement}

Arianna Schiano Lomoriello: Methodology, Data Curation, Formal analysis, Investigation, Visualization, Writing - Original Draft

Giulio Caperna: Data Curation, Formal analysis, Writing - Original Draft

Elisa De Stefani: Writing - Review \& Editing

Pier Francesco Ferrari: Conceptualization, Writing - Review \& Editing

Paola Sessa: Conceptualization, Supervision, Writing - Original Draft, Writing - Review \& Editing 


\section{Competing financial interests}

The authors declare no competing financial interests. 


\section{Acknowledgments}

We would like to thank Dr. Avinash R. Vaidya for kindly providing us with the stimuli used in the present study (see Vaidya et al., 2013). We are really grateful to all patients and their families for their patience and their incredible efforts to help our research through the visits and long trips from all over Italy to reach the lab. We would also like to thank the Associazione Italiana Sindrome di Moebius for supporting our research. We thank Elena Casagrande, Benedetta Ciabattoni, Pietro Dicorato, Ilaria Lissandron, Lorena Sabrina Pometti and Martina Viani for their valuable contribution with the data collection. 